\title{
ON THE DEFINITION OF KAC-MOODY 2-CATEGORY
}

\author{
JONATHAN BRUNDAN
}

Abstract. We show that the Kac-Moody 2-categories defined by Rouquier and by Khovanov and Lauda are the same.

\section{INTRODUCTION}

Assume that we are given the following data:

- a (not necessarily finite) index set $I$;

- integers $a_{i j}$ for each $i, j \in I$ such that $a_{i i}=2, a_{i j} \leq 0$ for all $i \neq j$, and $a_{i j}=0$ if and only if $a_{j i}=0$;

- positive integers $d_{i}$ such that $d_{i} a_{i j}=d_{j} a_{j i}$ for all $i, j \in I$.

Thus $A=\left(a_{i j}\right)_{i, j \in I}$ is a symmetrizable generalized Cartan matrix. Set $d_{i j}:=-a_{i j}$ for short. Fix also the additional data:

- a complex vector space $\mathfrak{h}$;

- linearly independent vectors $\alpha_{i} \in \mathfrak{h}^{*}$ for each $i \in I$ called simple roots;

- linearly independent vectors $h_{i} \in \mathfrak{h}$ for each $i \in I$ such that $\left\langle h_{i}, \alpha_{j}\right\rangle=a_{i j}$.

Let $\mathfrak{g}$ be the associated Kac-Moody algebra with Chevalley generators $\left\{e_{i}, h_{i}, f_{i}\right\}_{i \in I}$ and Cartan subalgebra $\mathfrak{h}$. Let $P:=\left\{\lambda \in \mathfrak{h}^{*} \mid\left\langle h_{i}, \lambda\right\rangle \in \mathbb{Z}\right.$ for all $\left.i \in I\right\}$ be its weight lattice. In $[\mathbb{R}$, Rouquier has associated to $\mathfrak{g}$ a certain 2-category $\mathfrak{A}(\mathfrak{g})$, which we will denote instead by $\mathcal{U}(\mathfrak{g})$. It depends also on:

- a ground ring $\mathbb{k}$;

- units $t_{i j} \in \mathbb{k}^{\times}$for $i, j \in I$ with $i \neq j$ such that $t_{i j}=t_{j i}$ if $d_{i j}=0$;

- scalars $s_{i j}^{p q} \in \mathbb{k}$ for $i, j \in I$ and $0 \leq p<d_{i j}, 0 \leq q<d_{j i}$ such that $s_{i j}^{p q}=s_{j i}^{q p}$. (Often one assumes further that these parameters are homogeneous in the sense that $s_{i j}^{p q}=0$ unless $p d_{i}+q d_{j}=d_{i} d_{i j}$, but we do not insist on that here.) The following is the definition from $[\underline{\mathrm{R}}, \S 4.1 .3]$ formulated in diagrammatic terms.

Definition 1.1. The Kac-Moody 2-category $\mathcal{U}(\mathfrak{g})$ is the strict additive $\mathbb{k}$-linear 2-category with object set $P$, generating 1-morphisms $E_{i} 1_{\lambda}: \lambda \rightarrow \lambda+\alpha_{i}$ and $F_{i} 1_{\lambda}: \lambda \rightarrow \lambda-\alpha_{i}$ for each $i \in I$ and $\lambda \in P$, and generating 2-morphisms $x$ : $E_{i} 1_{\lambda} \rightarrow E_{i} 1_{\lambda}, \tau: E_{i} E_{j} 1_{\lambda} \rightarrow E_{j} E_{i} 1_{\lambda}, \eta: 1_{\lambda} \rightarrow F_{i} E_{i} 1_{\lambda}$ and $\varepsilon: E_{i} F_{i} 1_{\lambda} \rightarrow 1_{\lambda}$, subject to certain relations. To record these, we adopt a diagrammatic formalism like in [KL, representing the identity 2 -morphisms of $E_{i} 1_{\lambda}$ and $F_{i} 1_{\lambda}$ by ${ }^{\lambda+\alpha_{i} \uparrow \lambda}{ }_{i}^{\lambda}$ and $\lambda-\alpha_{i} \underset{i}{\downarrow} \lambda$, respectively, and the other generators by

$$
x=\uparrow_{i} \lambda, \quad \tau=\searrow_{i}^{\lambda}, \quad \eta=\bigcup_{\lambda}^{i}, \quad \varepsilon=\bigcap_{i}^{\lambda} .
$$

2010 Mathematics Subject Classification: 17B10, 18D10.

Research supported in part by NSF grant DMS-1161094. 
We stress that our diagrams are simply shorthands for algebraic expressions built by horizontally and vertically composing generators; they do not satisfy any topological invariance other than the "rectilinear isotopy" implied by the interchange law. First, we have the quiver Hecke relations:

$$
\begin{aligned}
& \gamma_{i}^{\lambda}-X_{i}^{\lambda}=\sum_{i}^{\lambda}-X_{i}^{\lambda}= \begin{cases}\uparrow_{i}^{\uparrow} \uparrow_{j} & \text { if } i=j, \\
0 & \text { otherwise }\end{cases}
\end{aligned}
$$

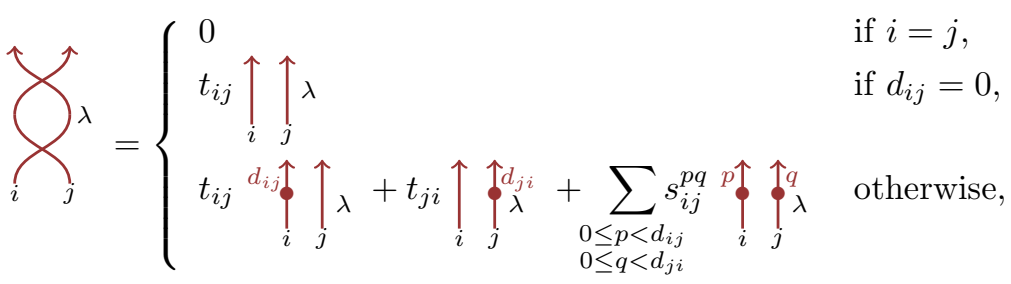

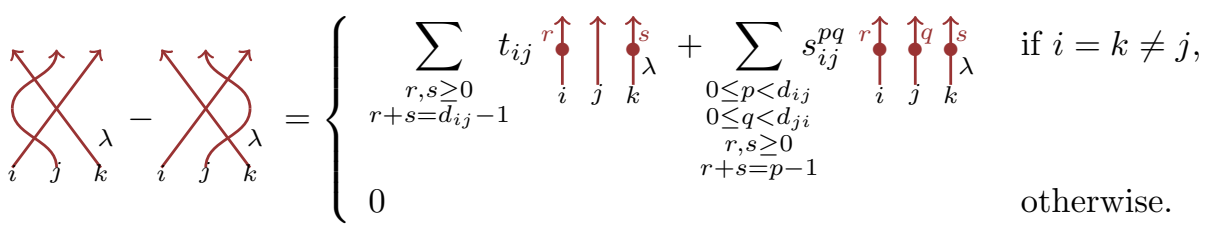

(Note in the above relations that we represent powers of $x$ by decorating the dot with a multiplicity.) Next we have the right adjunction relations

$$
\bigcap_{i} \uparrow_{\lambda}=\uparrow_{\lambda}, \quad \bigcup_{i} \bigcap_{\lambda}=\left.\right|_{\downarrow} ^{i} \lambda,
$$

which imply that $F_{i} 1_{\lambda+\alpha_{i}}$ is the right dual of $E_{i} 1_{\lambda}$. Finally there are some inversion relations. To formulate these, we first introduce a new 2-morphism

$$
\sigma=\searrow_{j}^{i}:=\bigcup_{\lambda} \searrow_{\lambda}: E_{j} F_{i} 1_{\lambda} \rightarrow F_{i} E_{j} 1_{\lambda} .
$$

Then we require that the following 2-morphisms are isomorphisms:

$$
\begin{aligned}
& \searrow_{j}^{i}: E_{j} F_{i} 1_{\lambda} \stackrel{\sim}{\rightarrow} F_{i} E_{j} 1_{\lambda} \\
& \searrow_{i}^{i} \oplus \bigoplus_{n=0}^{\left\langle h_{i}, \lambda\right\rangle-1} \stackrel{n}{i}_{i}^{\lambda}: E_{i} F_{i} 1_{\lambda} \stackrel{\sim}{\rightarrow} F_{i} E_{i} 1_{\lambda} \oplus 1_{\lambda}^{\oplus\left\langle h_{i}, \lambda\right\rangle} \quad \text { if }\left\langle h_{i}, \lambda\right\rangle \geq 0, \\
& \searrow_{i}^{i} \oplus \bigoplus_{n=0}^{-\left\langle h_{i}, \lambda\right\rangle-1} \bigcup_{\lambda}^{i} \hat{\boldsymbol{s}}_{n}: E_{i} F_{i} 1_{\lambda} \oplus 1_{\lambda}^{\oplus-\left\langle h_{i}, \lambda\right\rangle} \stackrel{\sim}{\rightarrow} F_{i} E_{i} 1_{\lambda} \quad \text { if }\left\langle h_{i}, \lambda\right\rangle \leq 0 .
\end{aligned}
$$

(This means formally that there are some additional as yet unnamed generators which serve as two-sided inverses to the 2-morphisms in (1.7)-(1.9).) 
Our main theorem identifies the 2-category $\mathcal{U}(\mathfrak{g})$ just defined with the KhovanovLauda 2-category from KL. Actually Khovanov and Lauda worked just with the choice of parameters in which $t_{i j}=1$ and $s_{i j}^{p q}=0$ always. Subsequently, Cautis and Lauda [CL generalized the definition to incorporate more general choices of these parameters as above. By the "Khovanov-Lauda 2-category" we really mean the more general version from $\mathrm{CL}$.

Main Theorem. Rouquier's Kac-Moody 2-category $\mathcal{U}(\mathfrak{g})$ is isomorphic to the Khovanov-Lauda 2-category.

The proof is an elementary relation chase. To explain the strategy, recall that the Khovanov-Lauda 2-category has the same objects and 1-morphisms as $\mathcal{U}(\mathfrak{g})$. Then there are generating 2-morphisms represented by the same diagrams as $x, \tau, \eta$ and $\varepsilon$ above, plus additional generating 2-morphisms $x^{\prime}: F_{i} 1_{\lambda} \rightarrow F_{i} 1_{\lambda}, \tau^{\prime}: F_{i} F_{j} 1_{\lambda} \rightarrow$ $F_{j} F_{i} 1_{\lambda}, \eta^{\prime}: 1_{\lambda} \rightarrow E_{i} F_{i} 1_{\lambda}$ and $\varepsilon^{\prime}: F_{i} E_{i} 1_{\lambda} \rightarrow 1_{\lambda}$ represented diagrammatically by

$$
x^{\prime}=\mathfrak{l}^{i} \lambda, \quad \tau^{\prime}=\searrow_{\lambda}^{i}, \quad \eta^{\prime}=\Upsilon_{\lambda}^{i}, \quad \varepsilon^{\prime}=\bigcap_{i}^{\lambda} .
$$

These satisfy further relations which we will recall in more detail later in the introduction. It is evident that all of the defining relations of $\mathcal{U}(\mathfrak{g})$ recorded above are satisfied in the Khovanov-Lauda 2-category. Hence there is a strict $\mathbb{k}$-linear 2 -functor from $\mathcal{U}(\mathfrak{g})$ to the Khovanov-Lauda 2-category which is the identity on objects and 1-morphisms, and maps the generating 2-morphisms $x, \tau, \eta$ and $\varepsilon$ to the corresponding 2-morphisms from $\mathrm{KL}, \mathrm{CL}$.

To see that this functor is an isomorphism, we construct a two-sided inverse. In order to do this, we need to identify appropriate 2-morphisms $x^{\prime}, \tau^{\prime}, \eta^{\prime}$ and $\varepsilon^{\prime}$ in $\mathcal{U}(\mathfrak{g})$ that will be the images of the additional generators (1.10) under the inverse functor. The definitions of $\eta^{\prime}$ and $\varepsilon^{\prime}$ that follow are essentially the same as Rouquier's "candidates" for second adjunction from [R, §4.1.4], except that we have renormalized by the sign $(-1)^{\left\langle h_{i}, \lambda\right\rangle+1}$ in order to be consistent with the conventions of $[\mathrm{KL}, \mathrm{CL}]$. We will also define a leftward crossing

$$
\sigma^{\prime}=\swarrow_{\lambda}^{i}: F_{i} E_{j} 1_{\lambda} \rightarrow E_{j} F_{i} 1_{\lambda},
$$

which we have chosen to normalize differently from the leftward crossing in [CL].

Definition 1.2. Define the downward dots and crossings $x^{\prime}$ and $\tau^{\prime}$ to be the right mates of $x$ and $\tau$ (up to the factor $t_{i j}^{-1}$ in the latter case):

$$
\begin{aligned}
& x^{\prime}=\downarrow^{i} \lambda:=\bigcup^{i} \oint \lambda, \\
& \tau^{\prime}=\searrow_{\lambda}^{i}:=t_{i j}^{-1} \bigcup_{\lambda} \stackrel{(1.6)}{=} t_{i j}^{-1} \overbrace{\lambda}^{j}
\end{aligned}
$$


Then to define $\eta^{\prime}, \varepsilon^{\prime}$ and $\sigma^{\prime}$, we assume initially that $\left\langle h_{i}, \lambda\right\rangle>0$. Thinking of (1.8) as a column vector of morphisms, its inverse is a row vector. We define the 2-morphisms $\sigma^{\prime}$ and $\eta^{\prime}$ so that $-\sigma^{\prime}$ is the leftmost entry of this row vector and $\eta^{\prime}$ is its rightmost entry:

$$
-\searrow_{i}^{i} \oplus \cdots \oplus \bigcup_{\lambda}^{i}:=\left(\searrow_{i}^{i} \oplus \cdots \oplus \varphi^{\left\langle h_{i}, \lambda\right\rangle-1} \varphi_{i}^{\lambda} \downarrow\right)^{-1} .
$$

Instead, if $\left\langle h_{i}, \lambda\right\rangle<0$, the morphism (1.9) is a row vector and its inverse is a column vector. We define $\sigma^{\prime}$ and $\varepsilon^{\prime}$ so that $-\sigma^{\prime}$ is the top entry of this column vector and $\varepsilon^{\prime}$ is its bottom one:

$$
-\nwarrow_{i}^{i} \oplus \cdots \oplus \bigcap_{i}^{\lambda}:=\left(\searrow_{i}^{i} \oplus \oplus \cdots \oplus \bigcup_{\lambda}^{i}-\left\langle h_{i}, \lambda\right\rangle-1\right)^{-1} .
$$

To complete the definitions of $\sigma^{\prime}, \eta^{\prime}$ and $\varepsilon^{\prime}$, it remains to set

$$
\begin{aligned}
& \nwarrow_{i}^{i}:=-\left(\searrow_{i}^{i} \lambda\right)^{-1} \quad \text { if }\left\langle h_{i}, \lambda\right\rangle=0, \\
& \bigcap_{i}^{\lambda}:=-\left\langle h_{i}, \lambda\right\rangle \bigcap_{\lambda} \quad \text { if }\left\langle h_{i}, \lambda\right\rangle \geq 0 \text {, } \\
& \bigcup_{\lambda}^{i}:=\bigodot_{-\left\langle h_{i}, \lambda\right\rangle}^{i} \lambda \quad \text { if }\left\langle h_{i}, \lambda\right\rangle \leq 0, \\
& \nwarrow_{j}^{i}:=\left(\searrow_{\lambda}^{i}\right)^{-1} \quad \text { if } i \neq j .
\end{aligned}
$$

Now to prove the Main Theorem we must show that all of the defining relations for the Khovanov-Lauda 2-category from [CL are satisfied by the 2-morphisms in $\mathcal{U}(\mathfrak{g})$ just introduced. First we will show that Lauda's infinite Grassmannian relation holds in $\mathcal{U}(\mathfrak{g})$. This asserts that, as well as the dotted bubble 2 -morphisms $r \bigcup_{i} \lambda, \lambda \bigcup_{i} s \in \operatorname{End}\left(1_{\lambda}\right)$ already defined for $r, s \geq 0$, there are unique dotted bubble 2-morphisms for $r, s<0$ such that

$$
\begin{gathered}
r \bigcup_{i}^{\lambda} \lambda=0 \text { if } r<\left\langle h_{i}, \lambda\right\rangle-1, \quad r \bigcup_{i}^{\lambda}=1_{1_{\lambda}} \text { if } r=\left\langle h_{i}, \lambda\right\rangle-1, \\
\sum_{\substack{r, s \in \mathbb{Z} \\
r+s=t-2}} s=0 \text { if } s<-\left\langle h_{i}, \lambda\right\rangle-1, \quad{ }_{i} s=1_{1_{\lambda}} \text { if } s=-\left\langle h_{i}, \lambda\right\rangle-1, \\
\lambda \bigcirc_{i}^{s}=0 \text { for all } t>0 .
\end{gathered}
$$


Using the infinite Grassmannian relation, we deduce that the inverses of the 2morphisms from (1.8) and (1.9) are

$$
-\searrow_{i}^{i} \oplus \bigoplus_{n=0}^{\left\langle h_{i}, \lambda\right\rangle-1} \sum_{r \geq 0} r \bigoplus_{i}^{\lambda}{ }_{-n-r-2}^{i}
$$

and

$$
-\searrow_{i}^{i} \oplus \bigoplus_{n=0}^{-\left\langle h_{i}, \lambda\right\rangle-1} \sum_{r \geq 0}{ }^{-n-r-2} \bigodot_{\bigcap_{i}^{i}}^{i},
$$

respectively. Several other of the Khovanov-Lauda relations follow from this assertion; see (3.16)-(3.18) below. After that, we will show that the 2-morphisms $\eta^{\prime}$ and $\varepsilon^{\prime}$ define a unit and a counit making the right dual $F_{i} 1_{\lambda+\alpha_{i}}$ of $E_{i} 1_{\lambda}$ also into its left dual:

$$
\uparrow \bigcap_{\lambda}=\uparrow_{\lambda}, \quad \bigcap \bigcup^{i} \lambda=\downarrow^{i} \lambda .
$$

Consequently, $\mathcal{U}(\mathfrak{g})$ is rigid, i.e. all of its 1-morphisms admit both a left and a right dual. Finally we will show that the other generating 2-morphisms are cyclic (up to scalars):

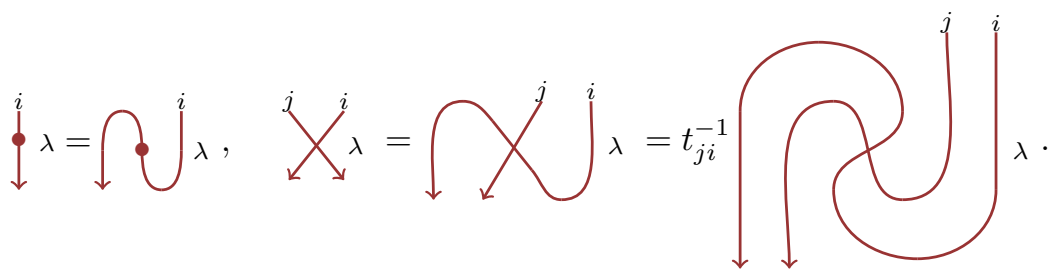

Acknowledgements. The diagrams in this paper were created using Till Tantau's TikZ package.

\section{Chevalley involution}

In this section we introduce a useful symmetry. First though we record some of the most basic additional relations that hold in the 2-category $\mathcal{U}(\mathfrak{g})$.

Lemma 2.1. The following relations hold:

$$
\begin{aligned}
& \bigcup_{\lambda}^{i}=\mathfrak{\iota}_{\lambda}, \\
& \bigcap_{i}^{\lambda}=\bigcap_{i}^{\lambda} \\
& \bigcap_{i}=\nearrow_{i} \searrow^{\lambda}, \\
& \chi_{j}^{i} \hat{\gamma}_{\lambda}=\bigcup_{j}^{i} . \\
& \chi_{i}^{\lambda}-\varnothing_{i}^{j}=\sum_{i}^{j}-\chi_{i}^{\lambda}= \begin{cases}\bigcup_{\lambda}^{j} & \text { if } i=j, \\
0 & \text { otherwise },\end{cases}
\end{aligned}
$$




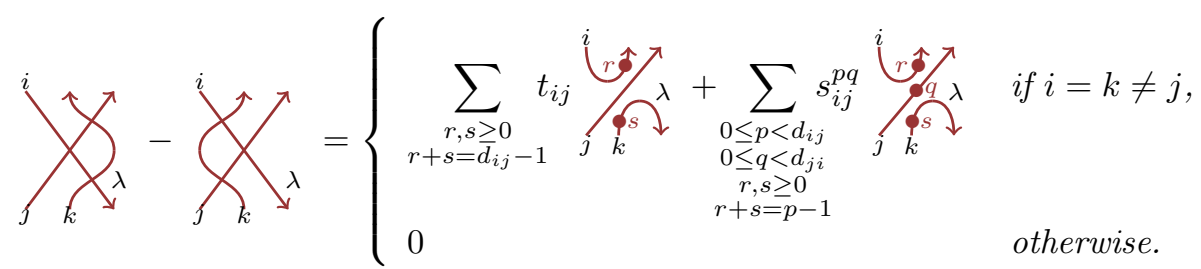

Proof. The first two relations follow from the definition (1.12) of the downward dot using the adjunction relations (1.5). The second two follow similarly from the definition of the rightward crossing (1.6). For (2.3), attach a rightward cap to the top right strand and a rightward cup to the bottom left strand of (1.2), then use (2.1) and the definition (1.6). Finally for (2.4), attach a rightward cap to the top right strand and a rightward cup to the bottom left strand in (1.4), then use (2.2) and the definition of the rightward crossing.

Taking notation from [CL, we define new parameters from

$$
{ }^{\prime} t_{i j}:=t_{j i}^{-1}, \quad{ }^{p} s_{i j}^{p q}:=t_{i j}^{-1} t_{j i}^{-1} s_{j i}^{q p} .
$$

The next lemma explains the significance of these scalars.

Lemma 2.2. The following relations hold:

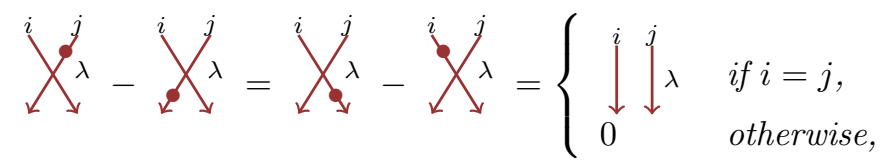

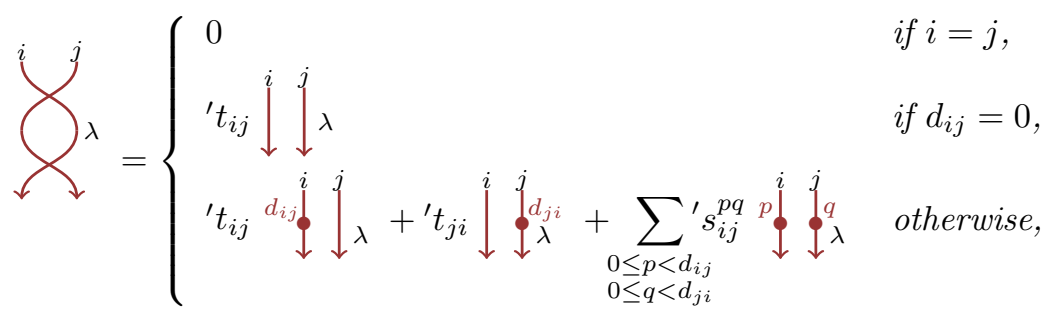

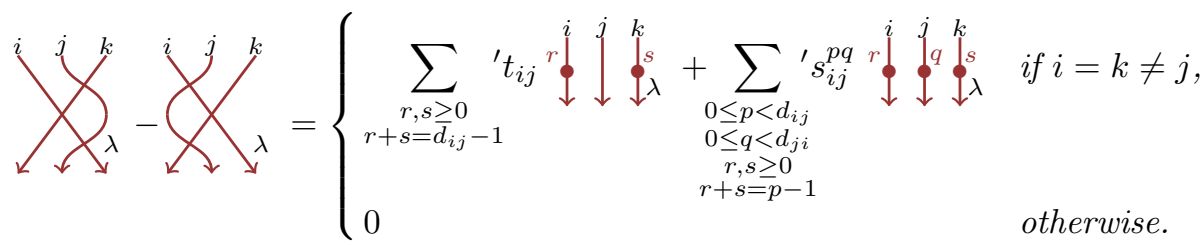

Proof. Put rightward caps on the top and rightward cups on the bottom of the relations (1.2)-(1.4), then use (1.5), the definitions (1.6), (1.12), (1.13), and (2.1)(2.2).

For any strict $\mathbb{k}$-linear 2-category $\mathcal{C}$, we write $\mathcal{C}^{\text {opp }}$ for the 2-category with the same objects as $\mathcal{C}$ but with morphism categories defined from $\mathcal{H}_{\mathrm{C}} \mathrm{m}_{\mathrm{Cop}}(\lambda, \mu):=$ $\mathcal{H o m}_{\mathcal{C}}(\lambda, \mu)^{\text {opp }}$, so the vertical composition in $\mathcal{C}^{\text {opp }}$ is the opposite of the one in $\mathcal{C}$, while the horizontal composition in $\mathcal{C}^{\text {opp }}$ is the same as in $\mathcal{C}$. 
Theorem 2.3. Let' $\mathcal{U}(\mathfrak{g})$ be the Kac-Moody 2-category defined as in Definition 1.1 but using the primed parameters from (2.5) in place of $t_{i j}$ and $s_{i j}^{p q}$. Then there is an isomorphism of strict $\mathbb{k}$-linear 2-categories

$$
\mathrm{T}: ' \mathcal{U}(\mathfrak{g}) \stackrel{\sim}{\rightarrow} \mathcal{U}(\mathfrak{g})^{\text {opp }}
$$

defined on objects by $\mathrm{T}(\lambda):=-\lambda$, on generating 1 -morphisms by $\mathrm{T}\left(E_{i} 1_{\lambda}\right):=F_{i} 1_{-\lambda}$ and $\mathrm{T}\left(F_{i} 1_{\lambda}\right):=E_{i} 1_{-\lambda}$, and on generating 2 -morphisms by:

$$
\hat{\uparrow}_{i} \lambda \mapsto \oint^{i}-\lambda, \quad \chi_{i} \mapsto-\searrow^{i}-\lambda, \quad \bigcup_{\lambda}^{j} \mapsto \bigcap_{i}^{-\lambda}, \quad \bigcap_{i}^{\lambda} \mapsto \bigcup_{-\lambda}^{i} .
$$

The effect of $\mathrm{T}$ on the other named 2-morphisms in ' $\mathcal{U}(\mathfrak{g})$ is as follows:

$$
\begin{aligned}
& \downarrow \lambda \mapsto \oint_{i}^{i}-\lambda, \\
& \searrow^{j} \lambda \mapsto-\searrow_{j}^{\nearrow}-\lambda, \\
& \bigcup_{\lambda}^{i} \mapsto \overbrace{i}^{-\lambda}, \\
& \bigcap_{i}^{\lambda} \mapsto \bigcup_{-\lambda}^{i}, \\
& \searrow_{i}^{j} \lambda \mapsto-t_{i j}^{-1} \searrow_{j}^{i}-\lambda \\
& \nwarrow_{i}^{j} \lambda \mapsto-t_{i j} \searrow_{j}^{i}-\lambda .
\end{aligned}
$$

Note in particular that $\mathrm{T}^{2}=\mathrm{id}$.

Proof. To see that $\mathrm{T}$ is well defined we need to verify that the images under $\mathrm{T}$ of the relations (1.2)-(1.5) and (1.7)-(1.9) with primed parameters hold in $\mathcal{U}(\mathfrak{g})^{\mathrm{opp}}$. For the first three, this follows from Lemma 2.2, while (1.5) is clear. For the remaining ones, we first note that $\searrow_{i}^{i} \mapsto-\searrow_{i}^{i} \chi_{-\lambda}$ by the definition (1.6). Then for example for (1.8), we must show for $\left\langle h_{i}, \lambda\right\rangle \geq 0$ that $\searrow_{i}^{i} \oplus \bigoplus_{n=0}^{\left\langle h_{i}, \lambda\right\rangle-1} \bigcup_{-\lambda}^{i} \hat{\gamma}_{n}$ is invertible in $\mathcal{U}(\mathfrak{g})$. This follows by composing (1.9) with $\operatorname{diag}(-1,1, \ldots, 1)$, using also (2.1). The rest of the theorem is a routine check from the definitions (1.12)(1.19).

We will often appeal to Theorem 2.3 to establish mirror images of relations in a horizontal axis. For example, applying it to (2.2), we obtain the following relation (which could also be deduced directly from the definition of the downward crossing):

Corollary 2.4. The following relations hold:

$$
\bigcup_{\lambda}^{i}=t_{j i} \chi^{j} \uparrow_{\lambda}, \quad \chi_{i}=t_{j i} \searrow_{i}^{j} .
$$

\section{The infinite Grassmannian Relation}

The goal in this section is to show that the infinite Grassmannian relation holds in $\mathcal{U}(\mathfrak{g})$. We begin by introducing notation for the other entries of the row vector 
that is the two-sided inverse of (1.8) and of the column vector that is the two-sided inverse of (1.9): let

$$
\begin{aligned}
& -\chi_{i}^{i} \oplus \bigoplus_{n=0}^{\left\langle h_{i}, \lambda\right\rangle-1} \bigoplus_{n}^{i} \lambda:=\left(\chi_{i}^{i} \oplus \bigoplus_{n=0}^{\left\langle h_{i}, \lambda\right\rangle-1}{ }_{i}^{n} \bigcap^{\lambda}\right)^{-1} \text { if }\left\langle h_{i}, \lambda\right\rangle \geq 0, \\
& \left.-\searrow_{i}^{i} \oplus \bigoplus_{n=0}^{-\left\langle h_{i}, \lambda\right\rangle-1} \downarrow^{n}\right\rangle_{i}^{\lambda}:=\left(\chi_{i}^{i} \oplus \bigoplus_{n=0}^{-\left\langle h_{i}, \lambda\right\rangle-1} \bigcup_{\lambda}^{i}\right)^{-1} \text { if }\left\langle h_{i}, \lambda\right\rangle \leq 0 .
\end{aligned}
$$

We will give a more explicit description of these 2-morphisms in (3.12)-(3.13) below. Note right away comparing the present definitions with (1.14)-(1.15) that

$$
\overbrace{\left\langle h_{i}, \lambda\right\rangle}^{i} \lambda=\uparrow \bigcup^{i} \lambda \text { if }\left\langle h_{i}, \lambda\right\rangle>0, \quad \overbrace{i}^{-\left\langle h_{i}, \lambda\right\rangle-1} \lambda=\bigcap_{i} \lambda \text { if }\left\langle h_{i}, \lambda\right\rangle<0 .
$$

Also for all admissible values of $n$ we have that

$$
\mathrm{T}\left(\uparrow_{n}^{i} \lambda\right)=\downarrow^{n} \stackrel{n}{i}^{-\lambda}, \quad \mathrm{T}\left(\downarrow^{n} \sum_{i}^{\lambda}\right)=\uparrow_{n}^{i}-\lambda,
$$

as follows on applying the isomorphism $\mathrm{T}$ from Theorem 2.3 to the definitions (3.1)(3.2) and using (2.1).

Lemma 3.1. The following relations hold:

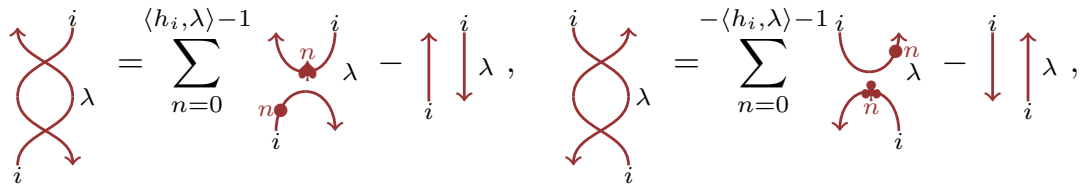

$$
\begin{aligned}
& \bigcup_{\lambda}^{i}=0, n \bigcap_{\lambda}=0,{ }^{n} \bigcup_{i}^{\lambda}=\delta_{n,\left\langle h_{i}, \lambda\right\rangle-1} 1_{1_{\lambda}} \text { all assuming } 0 \leq n<\left\langle h_{i}, \lambda\right\rangle \text {, } \\
& \bigcirc_{i}=0, \bigcirc_{\lambda}^{i}=0,{ }^{\lambda} \bigcirc_{i}^{n}=\delta_{n,-\left\langle h_{i}, \lambda\right\rangle-1} 1_{1_{\lambda}} \text { all assuming } 0 \leq n<-\left\langle h_{i}, \lambda\right\rangle \text {. }
\end{aligned}
$$

Proof. This follows from (3.1)-

The dotted bubbles ${ }^{r} \bigcup_{i} \lambda,{ }_{\lambda}{ }_{i}$ define endomorphisms of $1_{\lambda}$ for $r, s \geq 0$. We also give meaning to negatively dotted bubbles by making the following definitions 
for $r, s<0$ :

$$
\begin{gathered}
r \bigcup_{i} \lambda:=\left\{\begin{array}{cl}
-{ }_{\lambda}{ }^{i}{ }_{\lambda}^{-r-1}\left\langle h_{i}, \lambda\right\rangle & \text { if } r>\left\langle h_{i}, \lambda\right\rangle-1, \\
1_{1_{\lambda}} & \text { if } r=\left\langle h_{i}, \lambda\right\rangle-1, \\
0 & \text { if } r<\left\langle h_{i}, \lambda\right\rangle-1,
\end{array}\right. \\
{ }_{i} s:=\left\{\begin{array}{cl}
-\left\langle h_{i}, \lambda\right\rangle^{i} \lambda & \text { if } s>-\left\langle h_{i}, \lambda\right\rangle-1, \\
-s-1 & \text { if } s=-\left\langle h_{i}, \lambda\right\rangle-1, \\
1_{1_{\lambda}} & \text { if } s<-\left\langle h_{i}, \lambda\right\rangle-1 . \\
0 &
\end{array}\right.
\end{gathered}
$$

Note by Theorem 2.3, (2.1) and (3.4) that

$$
\mathrm{T}\left(r \bigcup_{i}^{\lambda}\right)=-\lambda \bigcup_{i} r, \quad \mathrm{~T}\left(\lambda \bigcup_{i} r\right)=r \bigcup_{i}-\lambda,
$$

for all $r, s \in \mathbb{Z}$. Given (3.6)-(3.9), the following theorem implies the infinite Grassmannian relation as formulated in formulae (1.20)-(1.22) in the introduction.

Theorem 3.2. The following holds for all $t>0$ :

$$
\sum_{\substack{r, s \in \mathbb{Z} \\ r+s=t-2}} r \bigcirc_{i} \lambda=0 .
$$

Proof. We prove this under the assumption that $\left\langle h_{i}, \lambda\right\rangle \geq 0$; the result when $\left\langle h_{i}, \lambda\right\rangle \leq 0$ then follows using Theorem 2.3 and (3.10). We have that

$$
\sum_{\substack{r, s \in \mathbb{Z} \\ r+s=t-2}} r \bigcup_{i} s \frac{3.8}{\underline{\underline{3.9}}} \sum_{n=0}^{\left\langle h_{i}, \lambda\right\rangle} n+t-1 \bigcup_{i} \lambda \bigcup_{i} n-1+\sum_{\substack{r \geq-1, s \geq 0 \\ r+s=t-2}} r \bigcup_{i} \lambda \bigcup_{i}
$$

It just remains to show that the final expression here is zero. When $\left\langle h_{i}, \lambda\right\rangle>0$ this follows by (3.6) and (3.8). Finally if $\left\langle h_{i}, \lambda\right\rangle=0$ then

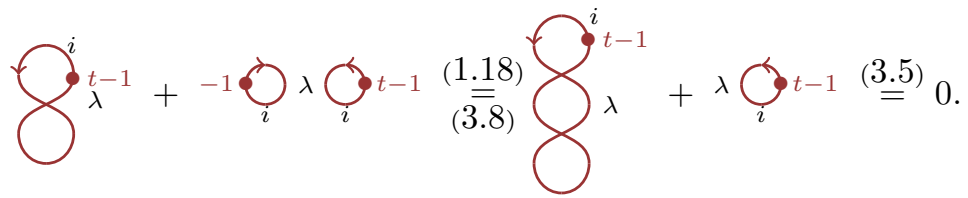


Corollary 3.3. The following relations hold:

$$
\begin{aligned}
& \left.\Upsilon_{n}^{i} \lambda=\sum_{r \geq 0} \int_{i}^{i}\right\}_{-n-r-2}^{i} \quad \text { if } 0 \leq n<\left\langle h_{i}, \lambda\right\rangle \text {, }
\end{aligned}
$$

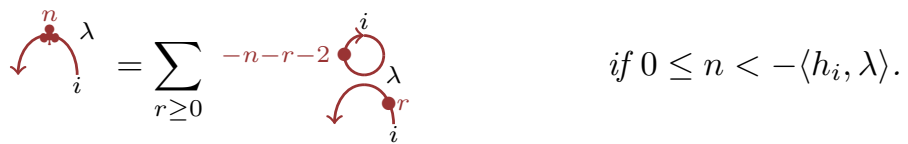

Proof. We explain the proof of (3.12); the proof of (3.13) is entirely similar. Remembering the definition (3.1), it suffices to show that the vertical composition consisting of (1.8) on top of (1.23) is equal to the identity. Using (3.5)-(3.6), this reduces to checking that

$$
\begin{array}{ll}
\sum_{r \geq 0} \sum_{r \geq 0}^{\lambda}=0 & \text { if } 0 \leq n<\left\langle h_{i}, \lambda\right\rangle, \\
m+r \bigcup_{-n-r-2}^{i} \overbrace{-n-r-2}^{i}=\delta_{m, n} 1_{1_{\lambda}} & \text { if } 0 \leq m, n<\left\langle h_{i}, \lambda\right\rangle .
\end{array}
$$

For (3.14), each term in the summation is zero: if $r \geq\left\langle h_{i}, \lambda\right\rangle$ the counterclockwise dotted bubble is zero by (3.9); if $0 \leq r<\left\langle h_{i}, \lambda\right\rangle$ we have that

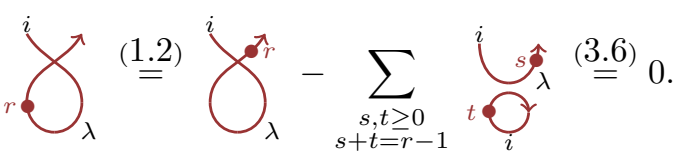

To prove (3.15), note by (3.6) and (3.9) that in order for $m+r \bigcup^{i}{ }^{i} \widehat{C}_{\lambda}-r-2$ to be non-zero we must have $m+r \geq\left\langle h_{i}, \lambda\right\rangle-1$ and $-n-r-2 \geq-\left\langle h_{i}, \lambda\right\rangle-1$. Adding these inequalities implies that $m \geq n$. Moreover if $m=n$ the only non-zero term in the summation is the term with $r=\left\langle h_{i}, \lambda\right\rangle-1-m$, which equals $1_{1_{\lambda}}$ by (3.6) and (3.9) again. Finally if $m>n$ the left hand side of (3.15) can be rewritten as

$$
\sum_{\substack{r, s \in \mathbb{Z} \\ r+s=m-n-2}} r \bigcup_{i} \times \bigcup_{i} s,
$$

which is zero by (3.11).

On substituting (3.12)-(3.13) into the definitions (3.1)-(3.2), this establishes the assertions about the 2-morphisms (1.23)-(1.24) made in the introduction.

Corollary 3.4. The following relations hold:

$$
\bigodot_{i}^{i}=\sum_{n=0}^{\left\langle h_{i}, \lambda\right\rangle-1} \sum_{r \geq 0} \underbrace{i}_{i} \bigodot_{i n-r-2}^{i}-\uparrow_{i}^{i} \downarrow^{i} \lambda,
$$




$$
\bigodot_{\lambda}^{i}=\sum_{n=0}^{-\left\langle h_{i}, \lambda\right\rangle-1} \sum_{r \geq 0}-n-r-2 \bigcup_{\substack{i \\ r}}^{i}-\downarrow_{i}^{i} \uparrow_{i} \lambda .
$$

Proof. Substitute (3.12)-(3.13) into (3.5).

Corollary 3.5. The following relations hold:

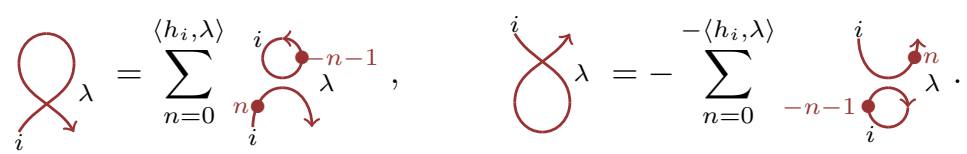

Proof. In view of Theorem 2.3 (2.1) and (3.10), it suffices to prove the left hand relation. We are done already by (3.7) if $\left\langle h_{i}, \lambda\right\rangle<0$. If $\left\langle h_{i}, \lambda\right\rangle \geq 0$ then:

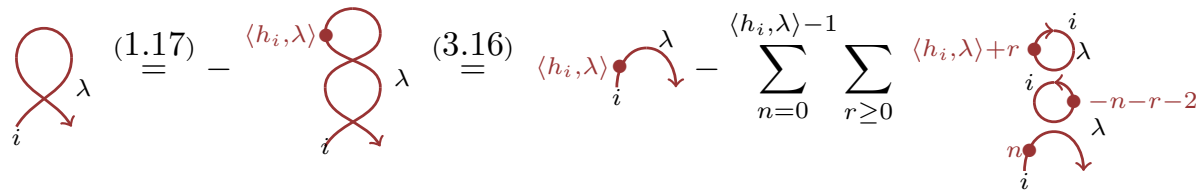

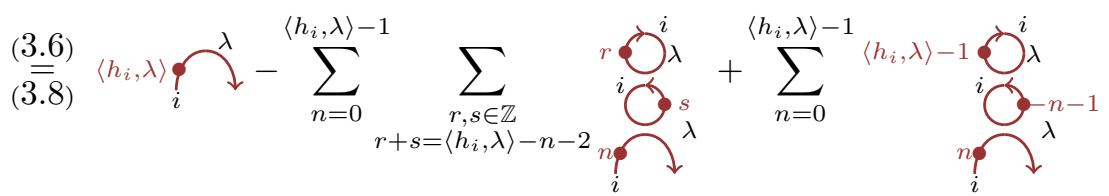

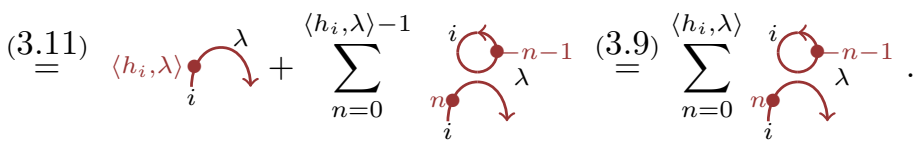

\section{LEFT ADJUNCTION RELATIONS}

In this section we show that the leftward cup and cap satisfy adjunction relations.

Lemma 4.1. The following relations hold:

$$
\begin{array}{ll}
\bigcap_{i}=\delta_{n,-\left\langle h_{i}, \lambda\right\rangle-2} \uparrow_{i}^{\lambda} & \text { if } 0 \leq n \leq-\left\langle h_{i}, \lambda\right\rangle-2, \\
\bigcap_{\lambda}=-\delta_{n,\left\langle h_{i}, \lambda\right\rangle} \uparrow_{i}^{\lambda} & \text { if } 0 \leq n \leq\left\langle h_{i}, \lambda\right\rangle .
\end{array}
$$

Proof. Proceed by induction on $n$. For the base case, convert the upward crossings to rightward ones using (1.5)-(1.6), apply (3.18) and (3.8)-(3.9), then invoke (1.5). For the induction step, pull a dot past the crossing using (2.1) and (1.2), then use (3.6) - (3.7) and the induction hypothesis. 
Lemma 4.2. Then the following relations hold:

$$
\begin{array}{ll}
\bigcap_{i}^{X_{i}}=\swarrow_{i}{ }_{i}^{\lambda} & \text { if }\left\langle h_{i}, \lambda\right\rangle<0, \\
\chi_{i}^{i}=\nearrow_{i}^{i} & \text { if }\left\langle h_{i}, \lambda\right\rangle>-2 .
\end{array}
$$

Proof. Let $h:=\left\langle h_{i}, \lambda\right\rangle$ for short. First we prove (4.3), so $h<0$. We claim that

$$
-\bigcap_{i}^{\lambda}=\uparrow_{i}^{\lambda} \bigcap_{i}^{\lambda}-\delta_{h,-1} \bigcap_{i} \uparrow_{i}^{\lambda}
$$

To establish the claim, we vertically compose on the bottom with the isomorphism $\uparrow_{i}^{i} \chi_{i} \oplus \bigoplus_{n=0}^{-h-1} \uparrow \bigcup_{i}^{i} \hat{i}_{n}$ arising from (1.9) to reduce to showing equivalently that

$$
\begin{aligned}
& -\bigotimes_{i}^{\top}=\bigcap_{i}^{-}-\delta_{h,-1} \bigcap_{i}^{\lambda}, \\
& -\bigodot_{i}^{i}=\uparrow_{i}^{i} \bigcirc_{\lambda}^{n}-\delta_{h,-1} \bigcap_{i} \text { for } 0 \leq n \leq-h-1 .
\end{aligned}
$$

Here is the verification of (4.6):

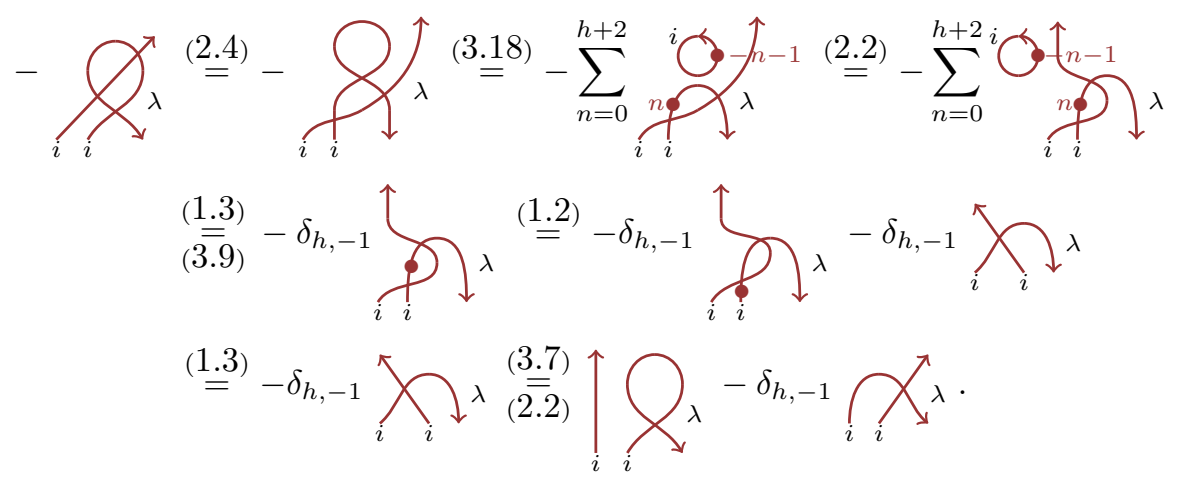

For (4.7), we note by (3.7) and (1.5) that the right hand side is equal to $\uparrow_{i} \lambda$ if $n=-h-1>0$, and it is zero otherwise. Now we simplify the left hand side:

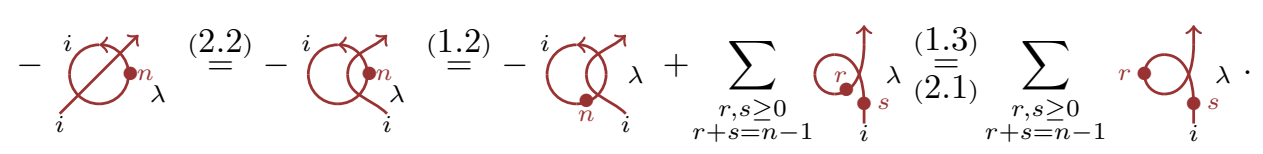

This is obviously zero if $n=0$. Assuming $n>0$, we apply (4.1) to see that it is zero unless $n=-h-1$, when the term with $r=-h-2, s=0$ contributes ${ }_{i}^{\uparrow \lambda}$. This completes the proof of the claim. Now to establish the relation (4.3), we vertically 
compose (4.5) on the bottom with $\searrow_{i}^{i} \uparrow_{i} \lambda$ to obtain the desired relation:

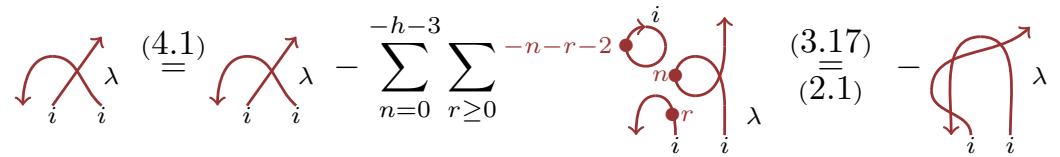

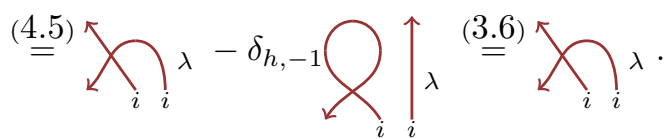

The proof of (4.4) follows by a very similar argument; one first checks that

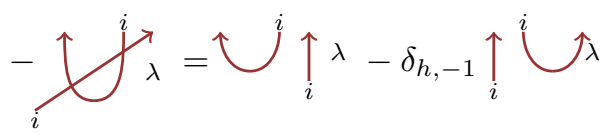

when $h>-2$ then vertically composes on the top with $\uparrow_{i} \swarrow_{i}^{i}$.

Theorem 4.3. The following relations hold:

$$
\uparrow \bigcap_{i}=\uparrow_{\lambda}, \quad \bigcap \bigcup^{i} \lambda=\left.\right|^{i} \lambda .
$$

Proof. It suffices to prove the left hand relation; the right hand one then follows using Theorem 2.3. Let $h:=\left\langle h_{i}, \lambda\right\rangle$ for short. If $h \geq 0$ then

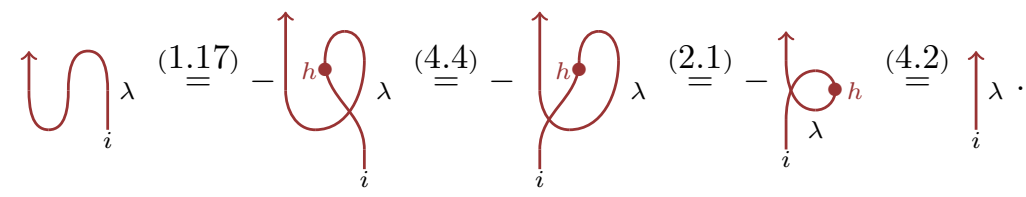

If $h \leq-2$ then

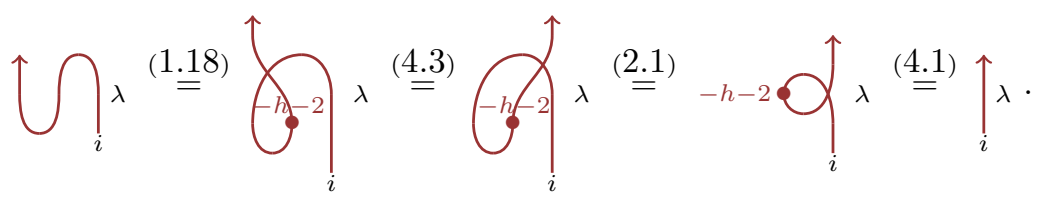

Finally if $h=-1$ then

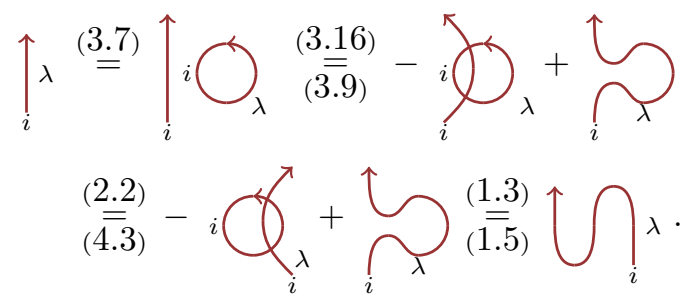

This completes the proof. 


\section{Cyclicity Relations}

At this point, the proof of the Main Theorem is reduced to checking the cyclicity relations. We proceed to do this.

Lemma 5.1. The following relation holds:

$$
\bigcup_{\lambda}^{i}=\uparrow \bigcup_{\lambda}^{i} \quad \text { if }\left\langle h_{i}, \lambda\right\rangle \geq 1 \text {. }
$$

Proof. The 2-morphisms on both sides of the desired identity go from $1_{\lambda}$ to $E_{i} F_{i} 1_{\lambda}$. To show that they are equal, we vertically compose them both on the top with the isomorphism (1.8) to reduce to proving instead that

$$
\bigcup_{\lambda} \oplus \bigoplus_{n=0}^{\left\langle h_{i}, \lambda\right\rangle-1}{ }^{n+1} \bigcup_{i} \lambda=\bigcup_{\lambda} \oplus \bigoplus_{n=0}^{\left\langle h_{i}, \lambda\right\rangle-1} \bigcup_{i}^{\lambda} .
$$

In these two column vectors of 2-morphisms, the entries involving dotted circles are equal thanks to (2.1). It just remains to observe that

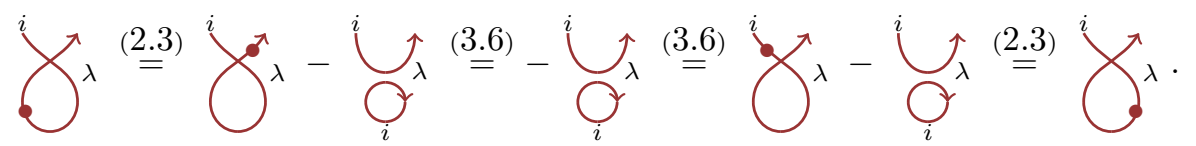

Lemma 5.2. Assuming that $i \neq j$, the following relations hold:

$$
\begin{array}{ll}
\nearrow_{i}^{\lambda}=t_{i j} \coprod_{j}^{\lambda} & \text { if }\left\langle h_{i}, \lambda\right\rangle<d_{i j}, \\
\chi_{\lambda}^{i}=t_{i j} \bigcup_{j}^{i} & \text { if }\left\langle h_{i}, \lambda\right\rangle \geq d_{i j} .
\end{array}
$$

Proof. Let $h:=\left\langle h_{i}, \lambda\right\rangle$ for short. First we prove (5.2) assuming that $h \leq 0$. By (1.7) and (1.9), the following 2-morphism is invertible:

$$
\searrow_{i}^{i} \oplus \bigoplus_{n=0}^{-h-1} \chi_{j}^{i} \hat{j}_{\lambda}^{n}
$$

Vertically composing with this on the bottom, we deduce that the relation we are trying to prove is equivalent to the following relations:

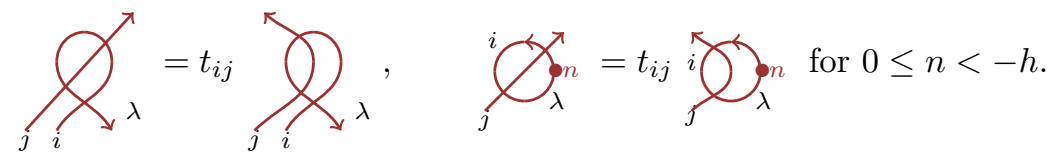

To establish the first of these, we pull the $j$-string past the $i i$-crossing:
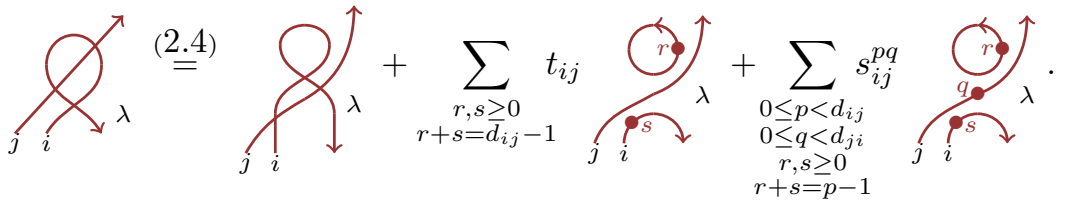
If $h<0$ then all the terms on the right hand side vanish thanks to (3.7). If $h=0$ and $d_{i j}>0$ everything except for the $r=d_{i j}-1$ term from the first sum vanishes, and we get $t_{i j} \uparrow_{j} \bigcap_{i}^{\lambda}$. Finally if $h=d_{i j}=0$, we only have the first term on the right hand side, which contributes $t_{i j} \uparrow \bigcap_{i}^{\lambda}$ again thanks to (3.18), (3.9), (2.2) and (1.3). This is what we want because:

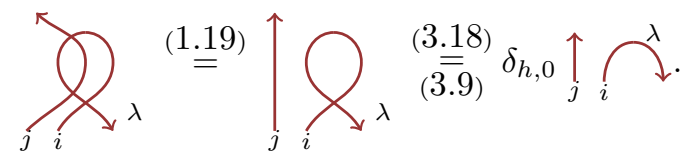

We are just left with the right hand relations from (5.4) involving bubbles:

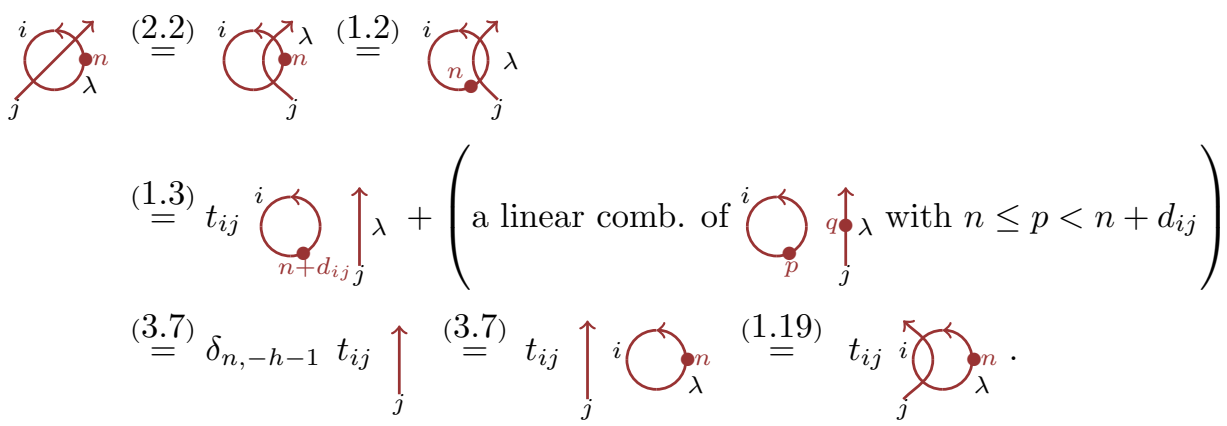

The relation (5.3) follows by a very similar argument to the one explained in the previous paragraph; the first step is to vertically compose on the top with the isomorphism

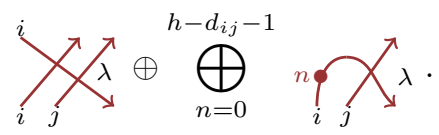

Finally we must prove (5.2) if $0<h<d_{i j}$. We vertically compose on the bottom with the isomorphism $\searrow_{j}^{i} \uparrow_{i} \lambda$ to reduce to proving that

$$
\bigcap_{i}^{\lambda}=t_{i j} \bigotimes_{j} \lambda
$$

To see this we apply (3.17) to transform the left hand side into

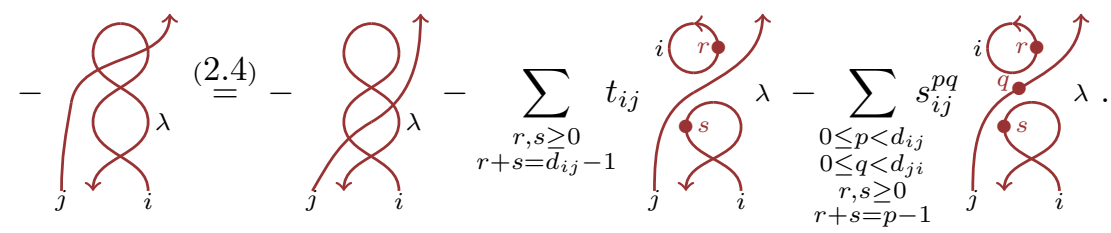

The first term on the right hand side here vanishes by (3.6). Also the terms in the summations are zero unless $r \geq d_{i j}-h-1$ and $s \geq h$ by (3.6)-(3.7), hence we are 
left just with the $r=d_{i j}-h-1, s=h$ term, which equals

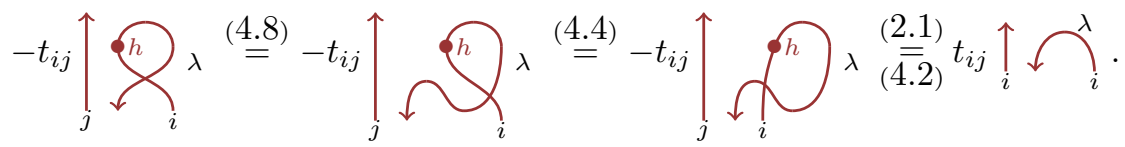

This is equal to the right hand side of (5.5) thanks to (1.19).

Theorem 5.3. The following relations hold for all $i, j \in I$ and $\lambda \in P$ :

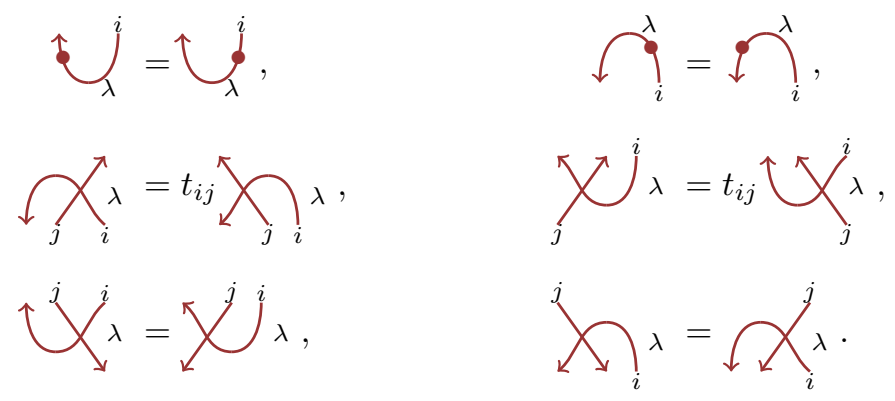

Proof. For (5.6), we already proved the left hand relation when $\left\langle h_{i}, \lambda\right\rangle \geq 1$ in (5.1). Now take this relation with $\lambda$ replaced by $\lambda+\alpha_{i}$, attach leftward caps to the top left and top right strands, then apply (4.8) to prove the right hand relation when $\left\langle h_{i}, \lambda\right\rangle \geq-1$. Finally apply Theorem 2.3 to the cases established so far to get the right hand relation when $\left\langle h_{i}, \lambda\right\rangle \leq-1$ and the left hand relation when $\left\langle h_{i}, \lambda\right\rangle \leq 1$.

The proofs of (5.7)-(5.8) follow by a similar strategy to the previous paragraph, starting from (4.3)-(4.4) and (5.2)-(5.3).

The final set of relations (1.26) needed to complete the proof of the Main Theorem follow easily from (5.6)-(5.8) using also (4.8).

\section{REFERENCES}

[CL] S. Cautis and A. Lauda, Implicit structure in 2-representations of quantum groups, to appear in Selecta Math..

[KL] M. Khovanov and A. Lauda, A diagrammatic approach to categorification of quantum groups III, Quantum Top. 1 (2010), 1-92.

[R] R. Rouquier, 2-Kac-Moody algebras; arXiv:0812.5023.

Department of Mathematics, University of Oregon, Eugene, OR 97403, USA

E-mail address: brundan@uoregon.edu 